\title{
Nano-optical concept design for light management
}

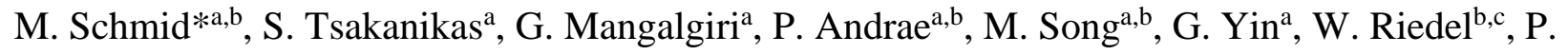 \\ Manley ${ }^{\mathrm{a}}$ \\ ${ }^{a}$ Nanooptical Concepts for PV, Helmholtz-Zentrum Berlin, Hahn-Meitner-Platz 1, 14109 Berlin, \\ Germany; ${ }^{b}$ Department of Physics, Freie Universität Berlin, Arnimallee 14, 14195 Berlin, Germany; \\ ${ }^{c}$ Heterogeneous Material System, Helmholtz-Zentrum Berlin, Hahn-Meitner-Platz 1, 14109 Berlin, \\ Germany
}

\begin{abstract}
Efficient light management in optoelectronic devices requires nanosystems where high optical qualities coincide with suitable device integration. The requirement of chemical and electrical passivation for integrating nanostrutures in e.g. thin film solar cells points towards the use of insulating and stable dielectric material, which however has to provide high scattering and near-fields as well. We investigate metal@dielectric core-shell nanoparticles and dielectric nanorods. Whereas core-shell nanoparticles can be simulated using Mie theory, nanorods of finite length are studied with the finite element method. We reveal that a metallic core within a thin dielectric shell can help to enhance scattering and near-field cross sections compared to a bare dielectric nanoparticle of the same radius. A dielectric nanorod has the benefit over a dielectric nanosphere in that it can generate much higher scattering cross sections and also give rise to a high near-field enhancement along its whole length. Electrical benefits of e.g. Ag@oxide nanoparticles in thin-film solar cells and $\mathrm{ZnO}$ nanorods in hybrid devices lie in reduction of recombination centers or close contact of the nanorod material with the surrounding organics, respectively. The optical benefit of dielectric shell material and elongated dielectric nanostructures is highlighted in this paper.
\end{abstract}

Keywords: core-shell nanoparticles, dielectric nanorods, scattering and absorption cross sections, near-field, Mie theory, finite element method, solar cells, hybrid devices

\section{INTRODUCTION AND MOTIVATION}

In recent years, nanostructures have attracted high interest due to their very particular material, thermal, electrical and optical properties. In the field of optics, there is a wide range of applications for nanostructures exploiting their plasmonic and photonic effects: spectroscopy and sensing benefit from signal enhancement by nanostructures [1], nanoparticle and photonic crystal waveguides use nanostructures for light guiding [2,3] and photovoltaics exploit absorption enhancement due to integrated nanostructures [4]. But what are the properties that make nanostructures such a unique optical system? Figure 1 highlights two specific characteristics of light absorption and scattering of an individual nanoparticle where the nanostructure outperforms a corresponding macrooptical system. A macroscopic lens can focus light incident onto its surface to a smaller area causing concentration, see Fig. 1b. The nanoparticle however, takes in light from an area larger than its geometrical cross section and concentrates it to a small area. A low coverage with nanoparticles may be sufficient for interaction with all light incident onto a surface. This phenomena of extinction cross sections larger than geometrical cross sections [5] is illustrated in Fig. 1a. Another aspect relates to the scattering of nanoparticles compared to bare refraction at an interface. Fig. 1d illustrates Snell's law for light passing an interface from low to high refractive index medium where even for large angles of incidence with respect to the normal, only a small angular spread can be obtained. In contrast, Fig. 1c represents the scattering distribution of a nanoparticle located at that same interface. At normal light incidence itself the nanoparticle can direct light to a much wider angular range, in particular also to angles larger than the angle of total reflection (expressed by the dashed line in Fig. 1c). This behavior leads to an enhancement of effective path length and in a suitable thin film system also to light trapping.

*martina.schmid@helmholtz-berlin.de; phone 4930 8062-43243; www.helmholtz-berlin.de

Optical Systems Design 2015: Optical Design and Engineering VI, edited by Laurent Mazuray,

Rolf Wartmann, Andrew P. Wood, Proc. of SPIE Vol. 9626, 96260E · C 2015

SPIE $\cdot$ CCC code: $0277-786 \mathrm{X} / 15 / \$ 18 \cdot$ doi: $10.1117 / 12.2191081$

Proc. of SPIE Vol. $962696260 \mathrm{E}-1$ 
a)

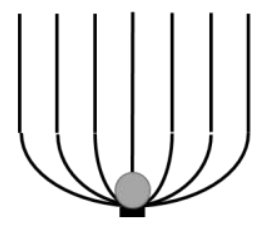

c)

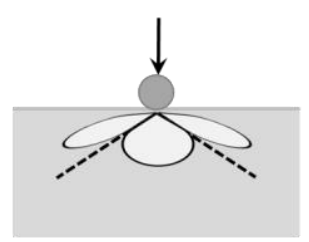

b)

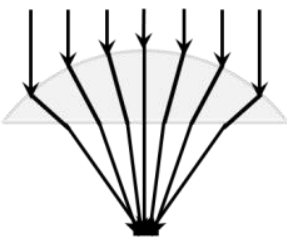

d)

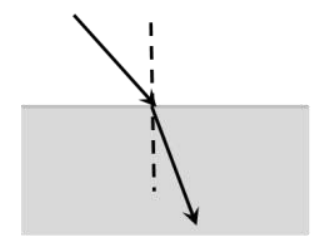

Figure 1. a) Schematic of a nanoparticle taking in light from an area larger than its geometrical cross section and focusing it; b) concentration behavior of a geometrical lens. c) Schematic of a nanoparticle at an interface scattering into angles larger than the angle of total reflection; d) Snell's law of refraction of light at an interface.

Many of the particular optical properties of nanoparticles have been studied in detail and it is not possible to give a comprehensive overview on all aspects covered in literature. We want to point out two geometries going beyond the basic nanoparticle structures and additionally them being relevant for applications. The first geometry is that of coreshell nanoparticles which brings out the benefit of chemical and electrical passivation of e.g. a metallic core in a dielectric shell. This passivation has proven to be of importance in systems where metallic nanoparticles would diffuse into the surrounding medium like $\mathrm{Ag}$ nanoparticles in $\mathrm{Cu}(\mathrm{In}, \mathrm{Ga}) \mathrm{Se}_{2}$ thin films [6]. In this example of thin-film photovoltaics; the added benefit of electrical passivation can be seen, since bare metallic nanostructures in the pnjunction can act as recombination centers reducing the solar cell efficiency. The second geometry we want to focus on is that of nanorods as an extreme of shape changes to dielectric nanoparticles. Nanorods are of high interest for those applications where a combined optical and electrical benefit is desired. To stay with the photovoltaics examples, we here mention nanowire solar cells where nanorods are made from absorber material and in addition to the optical benefits of light concentration a high carrier collection due to the radial junction is exploited. The material systems may be $\mathrm{Si}$ nanowire solar cells [7, 8] but also hybrid solar cells based on $\mathrm{ZnO}$ nanorods have gained attention [9]. In the following, we will firstly show the methods used for calculation of spherically symmetric and for other nanostructures. Then we will investigate core-shell particles and nanorods staying in the material system of $\mathrm{Ag}$ and intrinsic $\mathrm{ZnO}$. The central point we want to address is whether and when these more complicated nanostructures provide optical benefits in addition to their desired chemical and electrical properties.

\section{METHODS}

Analytical calculations usually give fast and exact results but are limited to simple structures of high symmetry. For more complicated structures, numerical methods are required which are generally applicable to any arbitrary geometry, yet at the cost of computational effort. We will briefly introduce Mie theory and the finite element method for nanostructure simulations and specify their application to the systems investigated here. A more detailed explanation about the two calculation methods was given in [10].

\section{Mie theory}

Mie theory describes absorption and scattering of light by a nanostructure by directly solving Maxwell's equations under consideration of a special geometry and related boundary conditions. Mie theory is therefore most popular for spherically symmetric structures where full analytic solutions exist [11]. Mie theory is an expansion of the scattered and internal fields of a spherical particle into the spherical vector harmonic basis functions, upon excitation by a plane wave. The weighting coefficients of the expansion are typically referred to as the Mie coefficients. A useful result from Mie theory is that the scattering and absorption cross sections, $\mathrm{C}_{\mathrm{sca}}$ and $\mathrm{C}_{\mathrm{abs}}$, of a particle depend only on the Mie coefficients and not on the basis functions themselves. $\mathrm{C}_{\mathrm{sca}}$ and $\mathrm{C}_{\mathrm{abs}}$ tell us how much of the light incident to the particle was scattered or 
absorbed. In the following we will refer to normalized cross sections Q (sometimes also denoted as efficiencies), which are given by the cross sections $\mathrm{C}$ divided by the particle geometrical cross section, i.e. $r^{2} \pi$ with $r$ the radius of the nanoparticle. These two normalized cross sections $\mathrm{Q}_{\text {sca }}$ and $\mathrm{Q}_{\text {abs }}$ relate to far field properties of the nanoparticle. In order to probe the near field properties, a definition for the near field cross section $\mathrm{Q}_{\mathrm{nf}}$ can also be found in the literature [12]. The near field cross section is the average value of the electric field squared at the particle's surface.

As mentioned above, Mie theory is mostly used for spherical nanoparticles. It can also be extended within the same formalism to core-shell nanoparticles [11] for which we apply it here. Infinite nanorods with cylindrical symmetry may also be calculated, however, Mie theory finds its limitations for more complicated shapes like finite nanorods or when non-homogeneous surroundings are considered. In these cases we have to move to a numerical method.

\section{Finite element method}

The finite element method (FEM) is a numerical method for solving partial differential equations such as Maxwell's equations [13]. The solutions to Maxwell's equations within a certain computational domain are expressed in terms of a set of basis functions. In order to describe the often complex behavior inside the domain, the total solution is formed from basis functions defined locally on so called individual domain elements. By reformulating Maxwell's equations in the weak formulation, simpler basis functions may be chosen, leading to the typical choice of polynomial functions. Given the boundary conditions at the edge of the computational domain, the total solution inside the domain can be directly solved for.

Due to the time-harmonic approach one wavelength can be calculated at a time and the simulation has to be repeated to obtain spectral information. Here we use the software package Comsol Multiphysics for FEM calculations of finite nanorods. The simulation geometry is a fix-sized box with perfectly matched layer boundaries within which the nanorod of variable height is centered. We used a two-step simulation, generating a background plane wave in the first step that is then used as the initial value for the second step to calculate absorption and scattering cross sections of the nanorod. Light is incident along the axis of the nanorod and the normalization of the cross sections through the cross sectional area therefore is by the same $r^{2} \pi$ as for the nanoparticle, $r$ is the radius of the nanorod here. Electric near-field distributions around the nanorod can also be extracted and are presented as normalized E-fields.

For calculation of the following results Ag refractive index data was taken from Palik [14], whereas $\mathrm{ZnO}$ optical constants were extracted from transmission and reflection measurements of a thin reference film using RefDex [15].

\section{RESULTS AND DISCUSSION}

For a basic understanding of optical properties of core-shell nanoparticles and nanorods, while also providing the link to materials relevant for applications, we chose as exemplary systems $\mathrm{Ag} @ \mathrm{ZnO}$ core-shell nanoparticles and $\mathrm{ZnO}$ nanorods and show simulation results obtained by Mie theory and FEM, respectively.

\section{Core-shell nanoparticles}

Silver is the most popular material for plasmonic nanoparticles due to low losses and a plasmonic resonance in the visible regime, therefore we use it as the core material in our investigations. To make a link to the $\mathrm{ZnO}$ nanorods following later, we choose $\mathrm{ZnO}$ as the shell material and investigate whether and how a Ag@ $\mathrm{ZnO}$ core-shell nanoparticle may outperform a bare $\mathrm{Ag}$ or $\mathrm{ZnO}$ nanoparticle. A comparison of metallic and dielectric nanoparticles has been carried out previously [10]. It was revealed that both material types can give rise to high scattering cross sections but the near-field distributions differ between homogeneous near-field enhancement around a metallic nanoparticle and strong forwards focusing for a dielectric one. In another investigation it was found that the choice of refractive index of the shell compared to the surrounding is crucial for efficient transition of near-fields [16]. Since for that the refractive index of the shell has to be higher than the one of the surrounding, we investigate our exemplary Ag@ZnO nanoparticles with a real part of the refractive index of $\mathrm{ZnO}$ of approximately 2 in a homogeneous surrounding of air. 


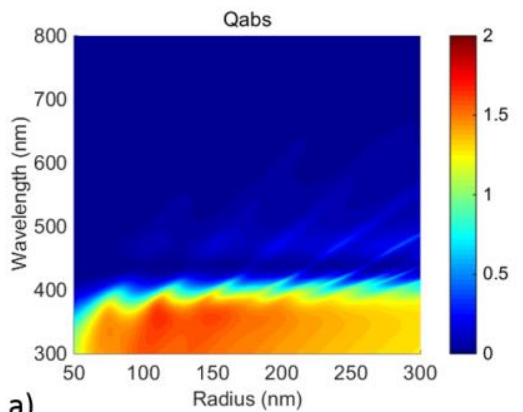

a)

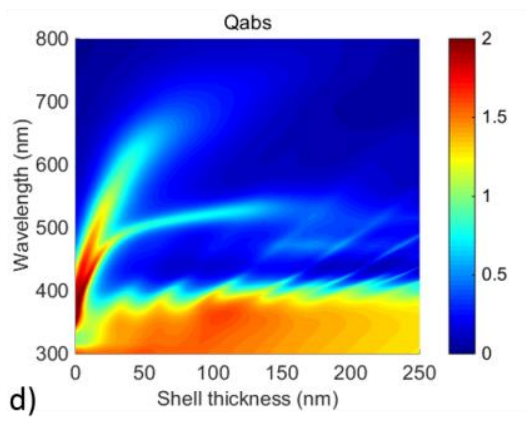

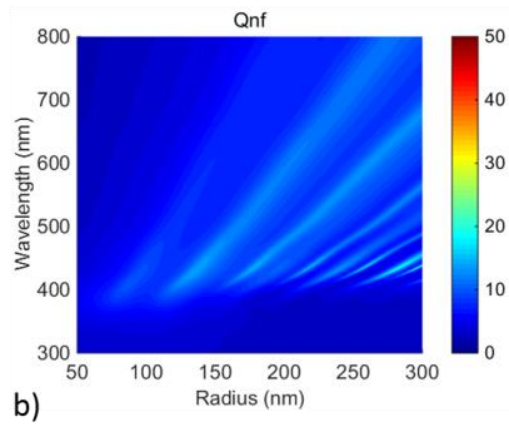

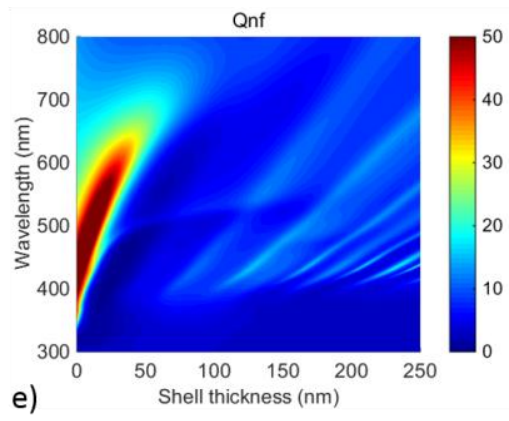

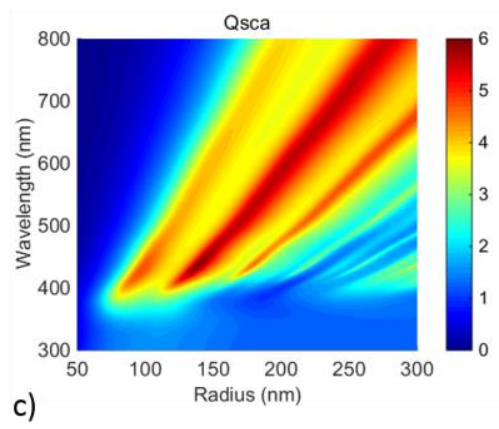

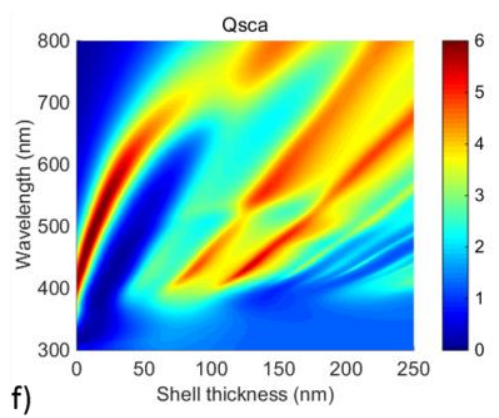

Figure 2. Normalized absorption, near-field and scattering cross sections of a) - c) a ZnO nanoparticle with increasing radius, d) - f) a Ag@ ZnO core-shell nanoparticle with core radius $50 \mathrm{~nm}$ and increasing shell thickness; in air.

The simulation results for $\mathrm{Q}_{\mathrm{abs}}, \mathrm{Q}_{\mathrm{nf}}$ and $\mathrm{Q}_{\mathrm{sca}}$ for the $\mathrm{Ag} @ \mathrm{ZnO}$ nanoparticle are depicted in Fig. $2 \mathrm{~d}-\mathrm{f}$, respectively, and compared to the values for a bare $\mathrm{ZnO}$ nanoparticle in Fig. 2 a-c. The cross sections are given as a function of both wavelength (y-axis) and radius (bare $\mathrm{ZnO}$ nanoparticle) or shell thickness ( $\mathrm{Ag} @ \mathrm{ZnO}$ nanoparticle). The $\mathrm{Ag}$ core has a radius of $50 \mathrm{~nm}$ so that the given ranges of the two $\mathrm{x}$-axes correspond to the same total particle radius. From the comparison of Fig. 2a and 2d, the absorption in the wavelength range below $400 \mathrm{~nm}$ relates to the $\mathrm{ZnO}$ and its band gap absorption. The two additional branches occurring for the core-shell particle, however, originate from the resonances of the $\mathrm{Ag}$ core that become redshifted in the increasing surrounding of $\mathrm{ZnO}$. The scattering visible for the $\mathrm{ZnO}$ nanoparticle in Fig. 2c also appears for the core-shell particle for shell thickness of above $150 \mathrm{~nm}$, as can be seen from Fig. 2f. In this case, the core-shell nanoparticle predominantly behaves like the dielectric one. For lower shell thicknesses, the strong influence of the metallic core dominates. Up to $50 \mathrm{~nm} \mathrm{ZnO}$ thickness, a strong scattering peak is present for the coreshell particle, whereas the bare dielectric particle shows almost no scattering for the same radius. A comparison to the scattering cross section of a bare $\mathrm{Ag}$ nanoparticle with $50 \mathrm{~nm}$ radius (not shown here) confirms these are the plasmonic modes of the core, which are shifted in the higher refractive index surrounding of the $\mathrm{ZnO}$. In case of medium sized shell thicknesses (around $100 \mathrm{~nm}$ ) the core-shell particles show no significant benefit until they start behaving like bare dielectric particles for shell thicknesses above $200 \mathrm{~nm}$. To achieve scattering cross sections as large as observed for $50 \mathrm{~nm}$ radius $\mathrm{Ag}$ nanoparticles with a $10 \mathrm{~nm}$ thick $\mathrm{ZnO}$ shell, bare $\mathrm{ZnO}$ particles with radii above $150 \mathrm{~nm}$ would be required. Most prominently, the metallic core fundamentally changes the near-field cross section of the core-shell compared to the dielectric nanoparticle (Fig 2e compared to 2b). In the presence of the Ag core a near-field cross section of more than 50 times the geometrical cross section occurs which is most pronounced for shell thicknesses of few $\mathrm{nm}$ only. When the $\mathrm{ZnO}$ shell thickness reaches $50 \mathrm{~nm}$ the prominent near-field has all but disappeared. Therefore, a $\mathrm{Ag}$ core shows a significant benefit to enhance in particular the near-field cross section when being inserted into a $\mathrm{ZnO}$ shell. The shell should only be a few tens of nanometers thick, otherwise the core-shell particle will behave mostly like a bare $\mathrm{ZnO}$ nanoparticle. Thus, embedding a metallic nanoparticle into a thin dielectric shell can help maintaining the high near-field enhancement of the metallic particle whilst achieving passivation and surpass the scattering and near-field of a bare dielectric nanoparticle. 


\section{Nanorods}

Nanorods are an interesting alternative to nanoparticles not only due to alternative applications but also from the perspective of improved optical properties. Here we want to make the transition from a structure with similar side lengths to an elongated one and consider absorption and scattering cross sections as well as near-field distributions. We simulate $\mathrm{ZnO}$ nanorods of $100 \mathrm{~nm}$ radius and a height ranging from $50 \mathrm{~nm}$ to $700 \mathrm{~nm}$ in surrounding medium of air. Fig. $3 \mathrm{a}$ and $3 \mathrm{~b}$ depict absorption and scattering cross section as a function of wavelength (y-axis) and nanorod height (x-axis; the denotation "height" is chosen since the nanorod is considered upright with light incident from the top). The absorption is limited to the wavelength range below $400 \mathrm{~nm}$, which is an intrinsic result of the absorption coefficient of $\mathrm{ZnO}$ and therefore independent of nanorod radius. The scattering cross section shows a maximum that is red-shifting with increasing height and centered between $500 \mathrm{~nm}$ and $600 \mathrm{~nm}$ wavelength. In contrast to absorption cross section, the scattering cross section experiences a red-shift with increasing nanorod radius (not shown here), which can be used to tune the resonance. We chose $\mathrm{ZnO}$ nanorods of $100 \mathrm{~nm}$ radius to obtain a high optical response in the visible wavelength range. The cross sections are increasing with increasing nanorod height due to the normalization with the areal cross section which remains constant despite increasing volume. In this way, a scattering cross section of up to 25 times the geometrical cross section has been observed for our $700 \mathrm{~nm}$ tall nanorods. When additionally dividing by the increasing height (without figure), the absorption cross section per unit length has a maximum for rods with heights comparable to the diameter. This may reflect that the short horizontal edges make the largest contribution to absorption. The scattering cross section per unit length, i.e. Qsca normalized to the nanorod heigth, however still keeps increasing as a function of height, implying that scattering relates to the increasing contribution of the vertical side length and less influence of the edges. In sum, tall rods are very beneficial for high scattering. An increasing scattering with increasing nanorod height and a red-shift of the resonance with increasing diameter are also in agreement with experimental findings reported in literature [17].
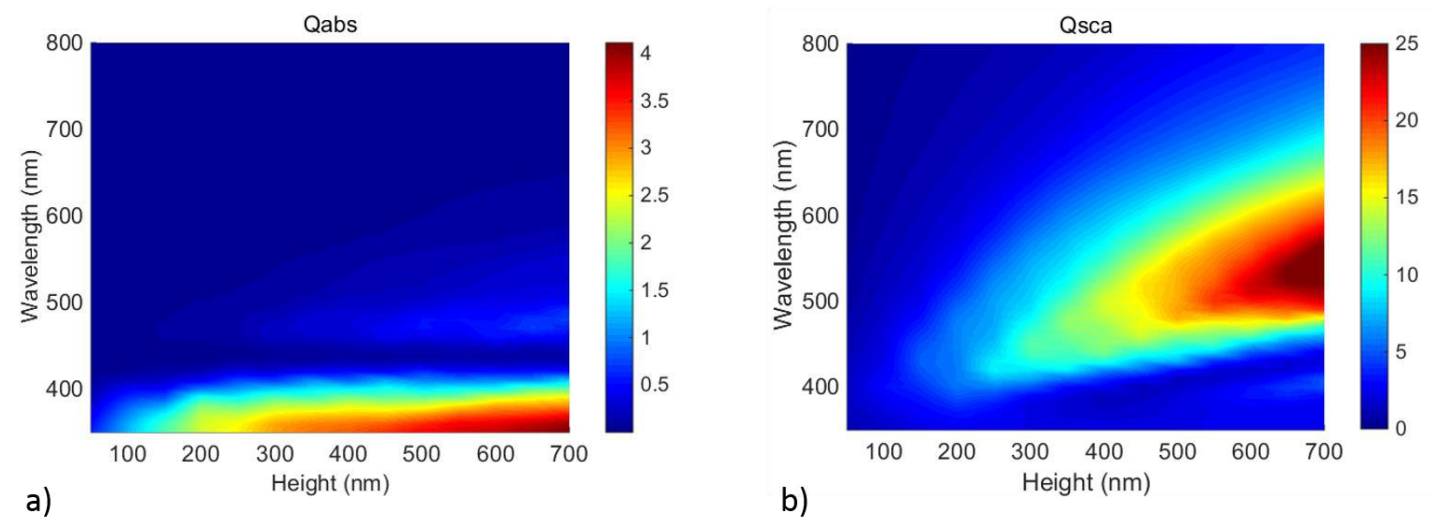

Figure 3. a) Normalized absorption and b) scattering cross sections of a $\mathrm{ZnO}$ nanorod (radius $100 \mathrm{~nm}$, illuminated with light incident along its axis) as a function of wavelength and nanorod height; in air.

Now we want to take a look at the electric near-field distribution around the nanorods. Fig. 4 shows the electric nearfield distribution for the $\mathrm{ZnO}$ nanorod of $100 \mathrm{~nm}$ radius with light incident from above and the normalized electric field being depicted in the plane of polarization. A wavelength of $600 \mathrm{~nm}$ was chosen due to a high optical response around that value according to Fig. 3b. The near-field of the nanorod in Fig. 4a with height equal to diameter (and therefore a square when viewed in cross section) resembles the distribution known from a disc [18]. When the height equals $300 \mathrm{~nm}$, i.e. half the wavelength, one loop like structure is visible inside the nanorod and a strongly enhanced near-field along the whole surface is observed (Fig. 4b). Maxima and minima emerging in nanorods with increasing height were also described by [19]. For a height equal to the full wavelength, i.e. $600 \mathrm{~nm}$ as shown in Fig. 4e, two loops are observed and maxima in near-field can be found along the whole nanorod. For heights other than a multiple of half the wavelength, however, part of the nanorod appears dark, meaning this area does not effectively contribute to near-field enhancement, see Fig. 4c,d,f. The longer the rod becomes, the more modes can fit and an increasingly homogeneous high near-field distribution around the nanorod emerges, as is confirmed by literature for very tall rods [20,21]. Combining the results from near-field investigations with the findings related to scattering cross sections, we can say that tall rods are very beneficial both for high scattering as well as for strongly enhanced near-fields along the whole surface. 


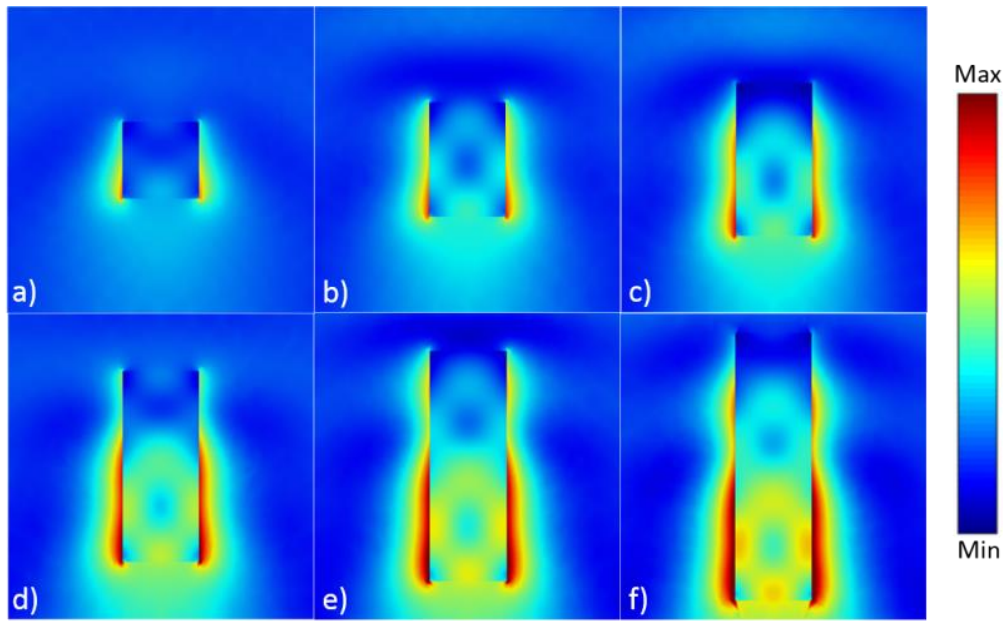

Figure 4. Normalized electric near-field distribution around a $\mathrm{ZnO}$ nanorod (radius $100 \mathrm{~nm}$ ) for light of $600 \mathrm{~nm}$ wavelength incident along the nanorod and depicted in the plane of polarization for a) $200 \mathrm{~nm}$, b) $300 \mathrm{~nm}$, c) $400 \mathrm{~nm}$, d) $500 \mathrm{~nm}$, e) 600 $\mathrm{nm}, \mathrm{f}) 700 \mathrm{~nm}$ nanorod height.

\section{SUMMARY AND APPLICATIONS}

In this paper we have investigated $\mathrm{Ag} @ \mathrm{ZnO}$ metal in dielectric core-shell nanoparticles using Mie theory and dielectric $\mathrm{ZnO}$ nanorods with the finite element method.

Dielectric material may be beneficial in terms of chemical and electrical passivation with respect to applications in optoelectronic devices. A dielectric shell with thickness up to a few tens of nanometers surrounding a metallic nanoparticle can also lead to an optical benefit. We have shown that it can enhance scattering cross sections compared to a purely dielectric nanoparticle and additionally give rise to a strong near-field cross sections. Integrating metal@dielectric core-shell structures into e.g. thin-film solar cells can allow to place the nanoparticles directly into the absorber. The direct contact to the absorber materials promises the highest absorption and hence performance enhancement. By the right choice of materials chemical stabilization is assured and electrical recombination omitted due to the passivating shell. In particular for $\mathrm{Cu}(\mathrm{In}, \mathrm{Ga}) \mathrm{Se}_{2}$ solar cells where the uncontrolled diffusion of metallic nanoparticles is highly critical, core-shell structures are a promising alternative. For a successful implementation, the requirement of refractive index of the shell higher than the one of surrounding material, which is approximately 3 in the example above, has additionally to be addressed which leads us towards shell materials such as AlSb [16].

The choice of purely dielectric nanostructures can be a favorable alternative. In order to go beyond the scattering cross sections of a nanosphere, nanorods with increasing height can be chosen. Their resonance can be well adjusted by the nanorod diameter in order to match the desired wavelength range. Compared to a dielectric nanosphere which shows pronounced forward scattering, the nanorod gives rise to high near-field enhancement along most of its surface. Maxima and minima can be identified according to the ratio of nanoparticle height and wavelength. The overall high near-field of dielectric nanorods can be exploited for applications where the nanorod is in direct contact with absorbing medium. An exploitation of a radial junction when applied to photovoltaics is an additional benefit. Hybrid devices where e.g. $\mathrm{ZnO}$ nanorods are additionally part of the photovoltaic junction are another application.

With core-shell nanoparticles and nanorods two types of nanostructures which can easily be derived from the nanosphere were shown. Their benefit for applications makes them good candidates for further detailed investigations.

\section{ACKNOWLEDGMENT}

Funding from the Helmholtz Association for the Young Investigator group VH-NG-928 within the Initiative and Networking fund is greatly acknowledged. 


\section{REFERENCES}

[1] K. M. Mayer, and J. H. Hafner, "Localized surface plasmon resonance sensors," Chemical Reviews, 111(6), 3828-3857 (2011).

[2] S. A. Maier, P. E. Barclay, T. J. Johnson et al., "Low-loss fiber accessible plasmon waveguide for planar energy guiding and sensing," Applied Physics Letters, 84(20), 3990-3992 (2004).

[3] E. Yablonovitch, "Photonic Crystals: Semiconductors of Light," Scientific American, 285, 47-55 (2001).

[4] M. A. Green, and S. Pillai, "Harnessing plasmonics for solar cells," Nature Photonics, 6(3), 130-132 (2012).

[5] C. F. Bohren, "How can a particle absorb more than the light incident on it?," American Journal of Physics, 51(4), 323-327 (1983).

[6] G. Yin, A. Steigert, P. Andrae et al., "Integration of plasmonic Ag nanoparticles as a back reflector in ultra-thin $\mathrm{Cu}(\mathrm{In}, \mathrm{Ga}) \mathrm{Se}_{2}$ solar cells," Applied Surface Science, accepted (2015).

[7] M. Gharghi, E. Fathi, B. Kante et al., "Heterojunction silicon microwire solar cells," Nano Letters, 12(12), 6278-6282 (2012).

[8] C. Lin, and M. L. Povinelli, "Optical absorption enhancement in silicon nanowire arrays with a large lattice constant for photovoltaic applications," Optics Express, 17(22), 19371-19381 (2009).

[9] W. Riedel, S. Wiesner, D. Greiner et al., "Hybrid solar cells with ZnO-nanorods and dry processed small molecule absorber," Applied Physics Letters, 104(17), 173503 (2014).

[10] M. Schmid, P. Andrae, and P. Manley, "Plasmonic and photonic scattering and near fields of nanoparticles," Nanoscale Research Letters, 9, 50/1-50/9 (2014).

[11] C. F. Bohren, and D. R. Huffmann, [Absorption and Scattering of Light by Small Particles] Whiley (1998).

[12] B. J. Messinger, K. U. von Raben, R. K. Chang et al., "Local fields at the surface of noble-metal microspheres," Physical Review B, 24(2), 649-657 (1981).

[13] Andrei V. Lavrinenko, Jesper Lægsgaard, Niels Gregersen et al., [Numerical Methods in Photonics] CRC Press (2014).

[14] E. D. Palik, [Handbook of optical constants of solids] Academic Press (1985).

[15] G. Yin, P. Manley, and M. Schmid, "Influence of substrate and its temperature on the optical constants of $\mathrm{CuIn}_{1-\mathrm{x}} \mathrm{Ga}_{x} \mathrm{Se}_{2}$ thin films," Journal of Physics D: Applied Physics, 47(13), 135101 (2014).

[16] P. Manley, F. Schmidt, and M. Schmid, [Light Extraction from Plasmonic Particles with Dielectric Shells and Overcoatings] Optical Society of America (2013).

[17] R. Tena-Zaera, J. Elias, and C. Lévy-Clément, "ZnO nanowire arrays: Optical scattering and sensitization to solar light," Applied Physics Letters, 93(23), 233119 (2008).

[18] M. A. Otte, M. C. Estévez, L. G. Carrascosa et al., "Improved Biosensing Capability with Novel Suspended Nanodisks," The Journal of Physical Chemistry C, 115(13), 5344-5351 (2011).

[19] J. Dorfmüller, R. Vogelgesang, W. Khunsin et al., "Plasmonic Nanowire Antennas: Experiment, Simulation, and Theory," Nano Letters, 10(9), 3596-3603 (2010).

[20] M. Fu, L. Qian, H. Long et al., "Tunable plasmon modes in single silver nanowire optical antennas characterized by far-field microscope polarization spectroscopy," Nanoscale, 6(15), 9192-7 (2014).

[21] Z. Zhang, Z. Zhang, L. Zhang et al., "Electric field enhancements around the nanorod on the base layer," Optics Express, 19(8), 7274-7279 (2011). 